\title{
DEPRESSION, ANXIETY AND STRESS IN WOMEN FOLLOWING ACUTE CORONARY SYNDROME: IMPLICATIONS FOR SECONDARY PREVENTION
}

\section{Authors:}

Michelle DiGiacomo, BA, MHSc(Hons), $\mathrm{PhD}$

School of Nursing, University of Western Sydney \& Nursing Research Unit, Sydney West Area Health Service

Patricia M. Davidson, BA, RN, MEd, PhD

School of Nursing, University of Western Sydney \& Nursing Research Unit, Sydney West Area Health Service

Anita Vanderpluym, BN (Hons)

School of Nursing, Deakin University, Melbourne, Victoria/Box Hill Hospital Partnership

\section{Rose Snell}

School of Nursing, Deakin University, Melbourne, Victoria/Box Hill Hospital Partnership

Linda Worrall-Carter, $\mathrm{PhD}, \mathrm{BEd}$

School of Nursing, Deakin University, Melbourne, Victoria/Box Hill Hospital Partnership

\section{Address for correspondence and reprints:}

Dr Michelle DiGiacomo

Nursing Research Unit

University of Western Sydney \& Sydney West Area Health Service.

PO Box 533

Wentworthville NSW 2145

Australia

E mail: michelle_digiacomo@wsahs.nsw.gov.au

Telephone: +610298403631

Fax: +61029840 3629 


\begin{abstract}
Objective

To document incidence of depression, anxiety, and stress in women more than six months following an acute coronary syndrome.

\section{Design}

Participants were identified from a coronary care unit database. The Depression Anxiety Stress Scales 21 (DASS 21) was sent to potential participants via postal survey.

\section{Setting}

A metropolitan teaching hospital in Melbourne, Australia

\title{
Participants
}

The cohort of women was aged between 55 and 70 years. They had been admitted to hospital with a diagnosis of acute coronary syndrome (ACS) between 6 - 14 months prior to participating in this study.

\section{Main Outcome Measures}

Scores on Depression, Anxiety, and Stress Scale (DASS 21)

\section{Results}

Of the 117 posted questionnaires, thirty-nine women with a mean age of 63 (SD 4.97) responded to the survey, representing a response rate of $33.3 \%$. Most participants scored within normal levels of depression (66.7\%), anxiety (60.5\%), and stress (70.3\%), however, mild to extremely severe levels of each construct $(33.4 \%, 39.6 \%$, and $29.7 \%$, respectively) were found.

\section{Conclusions}

The reporting of elevated levels of depression, anxiety and stress in a subset of women more than six months following an ACS event underscores the importance of 
ongoing screening for risk factors impacting on psychological well-being and the inclusion of this information in education and counseling strategies in both the inpatient and outpatient settings. Based on these pilot data, consideration of a screening system in the immediate post discharge period for women at risk and an education or support service are recommended. 


\section{Depression, anxiety and stress in women following acute coronary syndrome: implications for secondary prevention}

\section{Introduction}

Cardiovascular disease (CVD) remains a major cause of disease burden in both men and women internationally ${ }^{1}$. It is the main cause of death in women from developed countries, though traditionally heart disease has been viewed as a male condition ${ }^{2-4}$. Coronary heart disease (CHD) is the most common form of CVD and frequently manifests as acute coronary syndrome (ACS) which includes a range of clinical conditions from angina pectoris to acute myocardial infarction (AMI). Often referred to as acute cardiac events, these grave conditions are a major burden on healthcare resources and individuals in industrialized countries ${ }^{5}$.

Although it is well established that biochemical and lifestyle factors, such as inactivity and smoking are risk factors for CHD, increasingly the link between psychological and social risk factors in the development and progression of the disease has been noted ${ }^{6}$. This paper will discuss the influence of depression, anxiety and stress on the aetiology and progression of heart disease and report findings of a pilot study to derive empirical data to inform education and counseling strategies. To date, the majority of psychological and social interventions in women have targeted the immediate post discharge phase ${ }^{7}$. There is little information available on the longterm adjustment of women to the acceptance of their illness and management. Psychosocial risk factors should be monitored in the same way as cholesterol, smoking, activity and other modifiable risk factors as part of a comprehensive secondary prevention risk strategy ${ }^{8}$. 
Cardiac rehabilitation (CR) programs provide important mechanisms to deliver effective rehabilitation strategies as well as secondary prevention following an acute cardiac event ${ }^{9}$. However, CR participation rates in women are low suggesting that these programs do not necessarily meet their needs ${ }^{10}$. It is possible that women do not receive information to inform them of the importance of psychological and social issues in modulating their recovery. These issues are of great importance in women as it has been reported that women often have worse outcomes than men ${ }^{11-12}$.

Although the evidence base for depression and cardiovascular disease is strong, the data for anxiety and stress in influencing health outcomes is not as compelling. This discrepancy may be due to issues related to psychometric testing and clustering of factors as discussed below.

\section{Depression}

There is an expanding literature relating psychosocial factors with the development of CHD and with adverse prognosis following an acute coronary event ${ }^{13}$. In large prospective national studies in the United States of America (USA), depression was associated with an increased risk of CHD incidence in both men and women ${ }^{14}$. Findings of a 13-year prospective study suggested that individuals with major depression had a 4.5 times greater risk of AMI compared with those with no history of depression ${ }^{15}$. Further, the Women's Health Initiative Observational Study showed a significant association between depression and cardiovascular disease (CVD) risk in

older women ${ }^{16}$. In women with no history of CVD, depression was an independent predictor of death from CHD (50\% more likely) ${ }^{16}$. 
It is estimated that 15 to $20 \%$ of individuals suffer depression following a cardiac event $^{14}$. Depressed patients experience more social problems over the first year of recovery from their cardiac events, report impaired quality of life, less adherence to treatment and lifestyle advice, and are slower to return to work as compared to nondepressed patients ${ }^{13,17}$. While it is generally accepted that depression alone warrants treatment, there is continued debate as to whether depression is a risk factor for future cardiac mortality and morbidity. The National Health and Nutrition Examination Survey (NHANES) showed depression to be associated with increased CHD mortality in men, but not in women ${ }^{13}$. In this study more severe levels of depression were related to non-fatal CHD events in women with no relationship to fatal $\mathrm{CHD}^{14}$.

Some studies have shown that depression following AMI is associated with increased risk of future cardiac events and cardiac mortality ${ }^{15}$, however a number of studies have not shown this relationship ${ }^{13}$. Posited reasons for this discrepancy in mortality findings involve changes in the definitions of ACS and issues in study design, including small sample sizes and under-powering ${ }^{13}$. The occurrence and manifestation of depression following ACS fluctuates during the months of recovery and may interact with other psychosocial factors such as relationship pressures and social support, both of which can vary over time and be impacted by onset of a patient's cardiac illness $^{18}$. Furthermore, it has been suggested that timing of assessment for depression can alter results. For example, studies finding an effect for depression typically assessed depression in the first two weeks post cardiac event, while others which had assessed depression after five months found no association ${ }^{19}$. 


\section{Clustering of risk factors}

Psychosocial and conventional risk factors often coexist. Depressed persons are likely to smoke and be physically inactive, thus leading to an overweight condition ${ }^{6,20}$. Other theories determining causes look at possible biological mechanisms explaining the links between CHD, depression, and CVD, including decreased autonomic regulation leading to a decreased heart rate and increased platelet dysfunction ${ }^{20}$. This cycle of events predisposes patients to vascular occlusion and $\mathrm{AMI}^{21}$. A clinical investigation found depression is not only a risk factor for CHD but is a predictor of post-AMI mortality in persons without high levels of social support ${ }^{18}$. This study included 906 participants of which $31.5 \%$ were women. Negative emotions can interfere with a patient's capacity to cope with their physical illness, thus implicating a reduced quality of life and susceptibility to unhealthy lifestyle behaviours ${ }^{22}$.

The Enhancing Recovery in Coronary Heart Disease Patients Study (ENRICHD) was a multi-center clinical trial which assessed whether treating depression and increasing social support immediately after acute MI reduces the risk of recurrent nonfatal infarction and death ${ }^{23}$. Depressed patients received $\mathrm{CBT}^{24-25}$ while those with low social support had detailed assessments of their social needs, relationships, and deficits. Counseling sessions were tailored to address needs by focusing on strengthening network ties to be more functional, supportive, and satisfying, or to create new relationships. Although this intervention did not have an impact on the composite primary outcomes of all cause mortality and nonfatal MI, the intervention did improve depression and social support. It was also suggested that treatment for depression and provision of social support be provided later in the recovery trajectory 
or for a duration longer than the first 6 months after an acute event. This result underscores the complexity of the interaction of biological, psychological, and social factors on the progression of CHD.

\section{Anxiety}

In addition to depression, anxiety is recognized as a potentially modifiable risk factor for CHD and contributes to poorer prognosis after acute coronary events ${ }^{26}$. The Normative Aging Study found that higher levels of anxiety were associated with nearly double the risk of fatal $\mathrm{CHD}^{26}$. A 20-year follow-up of women in the Framingham Study found that anxiety symptoms were linked to significantly increased risk of AMI and coronary-related death in homemakers ${ }^{27}$. Additionally, a study of 912 patients from Australia, South Korea, Japan, England, and USA found that anxiety was significantly higher in women than in men following an $\mathrm{AMI}^{28}$. Moser et $\mathrm{al}^{28}$ concluded that higher anxiety is a cause of poorer outcomes including further morbidity and higher rates of mortality post-AMI.

\section{Stress}

Workplace issues are prevalent in the literature on stress and heart disease. Kimivaki et $\mathrm{al}^{29}$ assessed 812 industrial employees who were free from cardiovascular diseases at baseline. Findings indicated that high job strain, high effort expended, and low reward increased risk of cardiovascular mortality. Turkoski ${ }^{30}$ established a link between high job strain and increased serum cholesterol and hypertension over a five year period. Despite these findings, a National Heart Foundation of Australia (NHFA) position states that there is insufficient evidence linking work-related stressors such as job strain, chronic life events, hostility, and anxiety disorders with 
$\mathrm{CVD}^{6}$. Personal or external acute life stressors, however, can trigger coronary events; for example, bereavement or an earthquake ${ }^{6,21}$.

Although unable to find a direct link between stress and hypertension, the American Heart Association guidelines for CVD prevention in women note that stress, particularly in the workplace, leads to maladaptive coping mechanisms ${ }^{2}$. Some maladaptive forms of stress coping mechanisms may contribute to an increased risk of $\mathrm{CHD}^{2}$. One example of this phenomenon can be seen in Lindquist et al's ${ }^{31}$ study of 654 employees, which investigated the role of work stress and coping mechanisms on blood pressure. Findings indicated that poor dietary habits, increased alcohol consumption, and inactivity, enacted coping mechanisms for work stress, mediated blood pressure elevation ${ }^{29}$.

As discussed above, depression, anxiety, and stress are recognized as risk factors for coronary heart disease that are potentially modifiable. As a consequence of a lack of clarity in the research, a survey was undertaken in female survivors of an ACS event to examine their self-reported levels of depression, anxiety, and stress and to inform a long term secondary prevention risk strategy for women.

\section{Method}

Sample

In order to obtain a sample remotely from an acute cardiac event, 117 women were identified from a large metropolitan public hospital coronary care unit database in Melbourne, Australia. Women were eligible if they had been admitted to hospital with a verified diagnosis of ACS within 6 to 14 months of the event, and were aged 
between 55 and 70 . This age group was targeted as previous international research has indicated they have poorer prognoses and higher rates of co-morbidities than their male counterparts following an ACS event ${ }^{32}$. Following on from advice from the ENRICHD study ${ }^{23}$, women within 6-14 months of the event were selected in order to allow for a period of normalisation and adjustment, particularly as this study focussed on the adjustment and recovery of women with an emphasis on stress, anxiety, and depression. Exclusion criteria included limited English vocabulary, refusal of consent, and cognitive impairment. Approval to undertake this study was obtained from the Eastern Health Human Research Ethics Committee and the Deakin University Research and Graduate Studies Committee.

\section{Instrument}

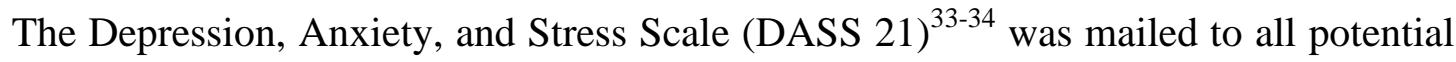
participants. The DASS 21 consists of three self-report scales designed to measure depression (dysphoria, hopelessness, devaluation of life, self-deprecation, lack of interest/involvement, anhedonia, and inertia), anxiety (autonomic arousal, skeletal muscle effects, situational anxiety, and subjective experience of anxious affect), and stress (chronic non-specific arousal, difficulty relaxing, nervous tension, and being easily upset/agitated, irritable/over-reactive and impatient $)^{33-34}$. The DASS has demonstrated high internal consistency and has been developed and validated in Australian populations. The alpha values for the 7 item scales are depression 0.81 ; anxiety 0.73; and stress 0.81. The DASS distinguishes well between aspects of depression, physical arousal, and psychological tension and agitation ${ }^{35}$. In a previous study using a normal population, reliability of the DASS was excellent and the measure possessed adequate convergent and discriminant validity ${ }^{36}$. The DASS 21 is 
particularly useful in that it is sensitive to symptoms of depression whereas tools such as the Beck Depression Inventory only identify depressive illness. Given the relationship of psychological and social factors to the outcomes of women with ACS, this instrument was considered appropriate to consider depression, anxiety, and stress as unique constructs.

\section{Procedure}

The hospital database was comprehensively searched and identified approximately 117 potential participants which met the inclusion criteria. These women were mailed an information sheet and the survey instrument. Potential participants were sent reply-paid envelopes to encourage all to respond and to prevent any respondent socioeconomic sample bias. In view of the fact that women were solicited from a database source, it was considered that methods to promote return of surveys such as mail reminders and follow-up surveys were inappropriate. Data was entered into SPSS (Version 12.0.1) and underwent descriptive analysis.

\section{Results}

\section{Sample Characteristics}

Within a six-week data collection timeframe, a response rate of $33.3 \%$ was achieved with thirty-nine women with a mean age of 63 (SD 4.97) years comprising the study sample. More than half of the women (53.8\%) reported a medical history of AMI. First diagnosis of heart disease occurred for $53.8 \%$ of the sample within twelve months prior to participating in the study.

\section{DASS Results}


Figure 1 represents the twenty-one items of the DASS and the corresponding percentages of women in the sample who indicated that statements either did not apply or applied to them in varying degrees. Figure 2 represents the women's levels of depression, anxiety, and stress by level of severity as indicated by their responses to the DASS items. Although most participants scored within normal levels of each construct, mild to extremely severe levels of depression (33.4\%), anxiety (39.6\%), and stress $(29.7 \%)$ were evident. Table 1 presents the DASS severity ratings and where our data fit into it.

\section{Depression Subscale}

As represented in Table 1, the sample achieved a mean score of 9.18 (SD 11.29). A normal score on the depression subscale falls between 0 and 9. This result indicates that study participants were on average bordering between normal and mild depression.

Responses to individual data items offer further insight into the nature of women's depression levels. In response to the statement regarding finding it difficult to work up the initiative to do things in the past week, $67 \%$ of women reported that this statement applied to them some, a good part, or most of the time (Fig. 1). In response to the statements, "I felt down-hearted and blue" and "I was unable to become enthusiastic about anything," $47 \%$ of the women indicated agreement.

Overall, responses to items within the depression subscale revealed the majority of women reported that depression-indicative statements did not apply to them, however between $28-67 \%$ of participants did reflect agreement to statements depicting 
depressive thoughts or feelings occurring in the previous week. These results suggest that the incidence of depression among women following diagnosis of heart disease is by no means insignificant and warrants attention.

\section{Anxiety Subscale}

Within the domain of anxiety, a score of 8-9 indicates mild anxiety and a score of 1014 indicates moderate anxiety (Table 1). The mean score of 9.26 (SD 10.54) indicates participants bordered on average between mild and moderate anxiety levels. Levels of anxiety (Fig. 2) depict the majority (60.5\%) of women within a normal range, however $21 \%$ were categorized as having severe or extremely severe levels of anxiety.

Again, responses to items within this subscale indicated the majority of women reported anxiety-indicative statements did not apply to them. However, more than half $(56 \%)$ of respondents confirmed that the statement, "I was aware of dryness of my mouth," applied to them some, a good part, or most of the time within the last seven days (Fig. 1). The remainder of the anxiety items reflected $20-47 \%$ of participants agreed to some degree with statements indicating the experience of anxiety.

\section{Stress}

Scores between 0 - 14 are classified as normal on the stress subscale. The sample achieved a mean score of 11.95 (SD 13.24), thereby indicating, on average, normal levels of stress experienced within this group (Table 1). Although participants again mainly reported normal levels of stress, nearly one third (29.7\%) of participants' 
scores indicated elevated stress levels (Fig. 2). More than half (64\%) of participants reported they had difficulty relaxing in the past seven days (Fig. 1). The remainder of items on this subscale indicated experiences of these circumstances in $40-51 \%$ of participants. These results indicate the pervasive experience of stress within this group of women following ACS.

\section{Discussion}

The pilot data reported above provides some insight into the experiences of depression, anxiety, and stress in the recovery period following an ACS event. The exploratory design of this pilot study does not allow us to comment on the attribution of these factors. The absence of pre-morbid baseline data also inhibits interpretation of these findings. It is important to be able to distinguish between intrinsic and extrinsic factors that modulate the recovery experience.

In the absence of expert assessment we cannot conclusively attribute a clinical diagnosis of depression or morbid levels of anxiety and/or stress. Despite this limitation, the data reveal that women continue to experience some distress. The study design disallowed deeper exploration of these factors and relationships to other factors such as health outcomes and symptomatology. It is possible that the small sample size, low response rate and ensuing responder bias may mean that these data are not representative of the total population. The limitations of small participant numbers also preclude the ability to generalize the study findings to other patient populations. Yet, these pilot data will be useful in powering future studies to decrease the burden of depression, stress, and anxiety in women and provide insight into the recovery trajectory of women. 
Despite these limitations, this select population's self-reported levels of depression, anxiety, and stress has significant implications for clinical management, ongoing secondary prevention initiatives, and patient education and counseling strategies. The increasing evidence-base for the impact of psychological and social factors highlight the importance of assessing and addressing these factors during and following the ACS admission.

\section{Implications for clinical practice and further research}

Although a low rate of psychological morbidity is reported in this sample, a substantive body of literature supports consideration of psychological factors in care planning. Clearly there is emerging data linking psychological factors and the progression of heart disease. These data suggest that screening and intervention programs need to target those at greatest risk. Consequently, further research is needed to identify interventions appropriate to patient characteristics, levels of distress, and the complex interplay of psychological constructs such as depression, stress, and anxiety, particularly among women who are at greatest risk. Information around the clustering of risk factors and symptoms, both psychological and physical, may assist in targeting high risk individuals. The configuration and timing of not only interventions but assessment windows is also poorly understood ${ }^{10,37-39}$.

The data derived from this study illustrate that although many women do not experience depression, stress, and anxiety at pathological levels, a significant number have ongoing distress which may potentially adversely impact on health outcomes ${ }^{7}$. The challenge is to implement reliable and valid measures to assess women at higher 
risk and ensure access to appropriate interventions. It is also important that this screening and vigilance continues beyond the acute care and rehabilitation sectors to primary care. It is possible that the use of valid and reliable measures in the acute care setting may assist at identifying those at greatest risk. Development of effective transitional care models involving practice nurses and general practitioners in these screening activities is likely to increase in importance particularly within a context of decreasing lengths of hospital stay ${ }^{39}$.

The development of a screening system and an education or support service for women post-ACS are possible interventions arising from this research that would benefit this population as a means of secondary prevention ${ }^{40}$. The reluctance for women to attend cardiac rehabilitation, in spite of higher levels of morbidity underscores the importance of analysis of the recovery trajectory of women following an ACS event and the tailoring of interventions to meet the needs of women at highest risk $^{7}$. It is also important that critical care nurses appreciate the importance of these factors when developing short and long term care plans for women with coronary heart disease $\mathrm{H}^{40}$. 


\section{References}

1. Australian Institute of Health and Welfare. Australia's Health 2002. Canberra: AIHW

2. Mosca LL, Benjamin AH, Berra EJ, et al. Physician awareness to cardiovascular disease prevention guidelines in the United States. Circulation. 2005; 111: 499-510.

3. Davidson PM, Daly J, Hancock K. Perceptions and experiences of heart disease: a literature review and identification of a research agenda in older women. Eur $J$ Cardiovasc Nurs. 2003; 2:255-264.

4. Worrall-Carter L, Jones T, Driscoll A. The experiences and adjustments of women following their first acute myocardial infarction. Contemp Nurse, 2005;19(1-2):211221.

5. Sinnaeve PR, Van de Werf FJ. Global patterns of health care for acute coronary syndromes. Curr Opin Cardiol. 2004; 19: 625-630.

6. Bunker SJ, Colquhoun DM, Esler MD, et al. "Stress" and coronary heart disease: psychosocial risk factors. Med J Aust. 2003; 178: 272-276.

7. Gallagher R, McKinley S , Dracup K. Effects of a telephone counselling intervention on psychosocial adjustment in women following a cardiac event. Heart \& Lung 2003; 32 (2): 79 - 87. 
8. National Heart Foundation Australia (NHFA) (2003). Reducing risk in Heart Disease: National Heart Foundation and The Cardiac Society of Australia and New Zealand.

9. Stephen J Bunker and Alan J Goble Cardiac rehabilitation: under-referral and underutilisationMJA 2003; 179 (7): 332-333

10. Gallagher R, McKinley S , Dracup K. Predictors of women's attendance at cardiac rehabilitation programs, Progress in Cardiovascular Nursing $2003: 18: 121$ 126.

11. Mertensson J, Karlson J, Fridlund B, 1998 Female patients with congestive heart failure: how they conceive their life situation. J Adv Nurs 28(6):1216-1224.

12. National Heart, Lung and Blood Institute (1998). The Framingham Heart Study, http://www.framingham.com/heart/index.htm, last accessed 10/06/04.

13. Steptoe A, Whitehead DL. Depression, stress and coronary heart disease: the need for more complex models. Heart. 2005; 91: 419-420.

14. Ferketich AK, Schwartzbaum JA, Frid DJ, Moeschberger ML. Depression as an antecedent to heart disease among women and men in the NHANES I study. Arch Intern Med. 2000; 160(9):1261-1268 
15. Pratt L, Ford D, Crum R, Armenian H, Gallo J, Eaton W. Depression, psychotropic medication, and risk of myocardial infarction. Circulation. 1996; 94 : 3123-3129.

16. Wassertheil-Smoller S, Shumaker S, Ockene J, et al. Depression and Cardiovascular Sequelae in Postmenopausal Women: The Women's Health Initiative (WHI). Arch Intern Med. 2004;164: 289-298.

17. Lett HS, Blumenthal JA, Babyak MA, et al. Depression as a risk factor for coronary artery disease: Evidence, Mechanisms, and Treatment. Psychosom Med, 2004;66: 305-315.

18. Frasure-Smith N, Lesperance F, Gravel G, et al. Social Support, Depression, and Mortality During the First Year After Myocardial Infarction. Circulation. 2000;101: 1919-1924.

19. Stewart RA, North FM, West TM, et al. Depression and cardiovascular morbidity and mortality: cause or consequence? Eur Heart J. 2003; 24: 2027-2037.

20. Katon WJ, Lin EHB, Russo J et al. Factors in Patients with Diabetes Mellitus and Major Depression. J General Intern Med. 2004;19:1192-1199.

21. Glassman AH. Depression and the course of coronary artery disease. Am J Psychiatry. 1998;155: 4-11. 
22. Lesperance F, Frasure-Smith, N. Negative emotions and coronary heart disease: getting to the heart of the matter. Lancet, 1996;347-414.

23. ENRICHD Investigators. Effects of treating depression and low perceived social support on clinical events after myocardial infarction. The Enhancing Recovery In Coronary Heart Disease Patients (ENRICHD) Randomised Trail. J Am Med Assoc. 2003;289 (23): 3106-3116.

24. Beck AT, Rush AJ, Shaw BF, Emery G. Cognitive Therapy of Depression. New York, NY: Guilford Press; 1979.

25. Beck J. Cognitive Therapy: Basics and Beyond. New York, NY: Guilford Press; 1995.

26. Kawachi I, Sparrow D, Vokonas PS, Weiss ST. Symptoms of anxiety and risk of coronary heart disease. The Normative Aging Study. Circulation, 1994;90: 22252229.

27. Eaker E, Pinsky J, Castelli W. Myocardial infarction and coronary death among women: psychosocial predictors from a 20-year follow-up of women in the Framingham Study. Am J Epidemiol. 1992; 135: 854-864.

28. Moser DK, Dracup K, McKinley S, et al. An international perspective on gender differences in anxiety early after acute myocardial infarction. Psychosom Med. $2003 ; 511-516$ 
29. Kivimaki M, Leino-Arjas P, Luukkonen R, Riihimaki H, Vahtera J, Kirjonen J. Work stress and risk of cardiovascular mortality: prospective cohort study of industrial employees. Br Med J, 2002; 325(7369): 857-860.

30. Turkoski. An ounce of prevention: Drugs used to treat hyperlipidemia (Part 1). Orthop Nurs. 2004; 23:58-61.

31. Lindquist TL, Beilin LJ, Knuiman MW. Influence of Lifestyle, Coping, and Job Stress on Blood Pressure in Men and Women. Hypertension. 1997;29: 1-7.

32. Vaccarino V, Krumholz HM, Yarzebski J, Gore JM, Goldberg R.J. Sex Differences in 2-year Mortality after Hospital Discharge for Myocardial Infarction. Ann Intern Med. 2001;134: 173-181.

33. Lovibond SH, Lovibond PF. Manual for the depression, Anxiety Stress Scales (DASS). Psychology Foundation Monograph. Sydney: The Psychology Foundation, University of New South Wales; 1993.

34. Lovibond SH, Lovibond PF. The structure of negative emotional states: Comparison of the Depression, Anxiety Stress Scales (DASS) with the Beck depression and anxiety inventory. Behav Res Therapy. 1995; 33: 335-343.

35. Antony MM, Bieling PJ, Cox BJ, Enns MW, Swinson RP. Psychometric properties of the 42-item and 21-item versions of the Depression Anxiety Stress 
Scales in clinical groups and a community sample. Psychol Assess, 1998; 102: 176181.

36. Crawford JR, Henry JD. The Depression Anxiety Stress Scales (DASS): Normative data and latent structure in a large non-clinical sample. Br J Clin Psychol. 2003;42: 111-131.

37. Hamel L, Oberle K. Cardiovascular risk screening for women. Clin Nurse Spec. 1996;10: 275-279.

38. Jacobs SK, Eckel RH. Evaluating and managing cardiovascular disease in women: Understanding a women's heart. Circulation. 2005;111: 383-384.

39. Halcomb EJ, Patterson E, Davidson PM. Evolution of practice nursing in Australia. Journal of Advanced Nursing 55(3): 376-390.

40. Goldston K, Davidson PM. On behalf of the NHFA/CSANZ Reducing Risk in Heart Disease Guidelines Working Group. Australian coronary heart disease secondary prevention guidelines: implications for nursing practice and research to improve cardiovascular health outcomes. Australian Nurses Journal 2003; Dec 03/Jan 04. Clinical Update. 
Table l. Subscale results according to DA SS Severity Ratings

\begin{tabular}{|l|c|c|c|}
\hline Normal & Depression & Anxiety & Stress \\
\hline Mild & $0-9$ & $0-7$ & $(0-14)$ \\
\hline Moderate & $10-13$ & $8-9$ & $15-18$ \\
\hline Severe & $14-20$ & $10-14$ & $19-25$ \\
\hline Extremely Severe & $21-27$ & $15-19$ & $26-33$ \\
\hline
\end{tabular}


Figure 1. Depression, Anxiety and Stress Items

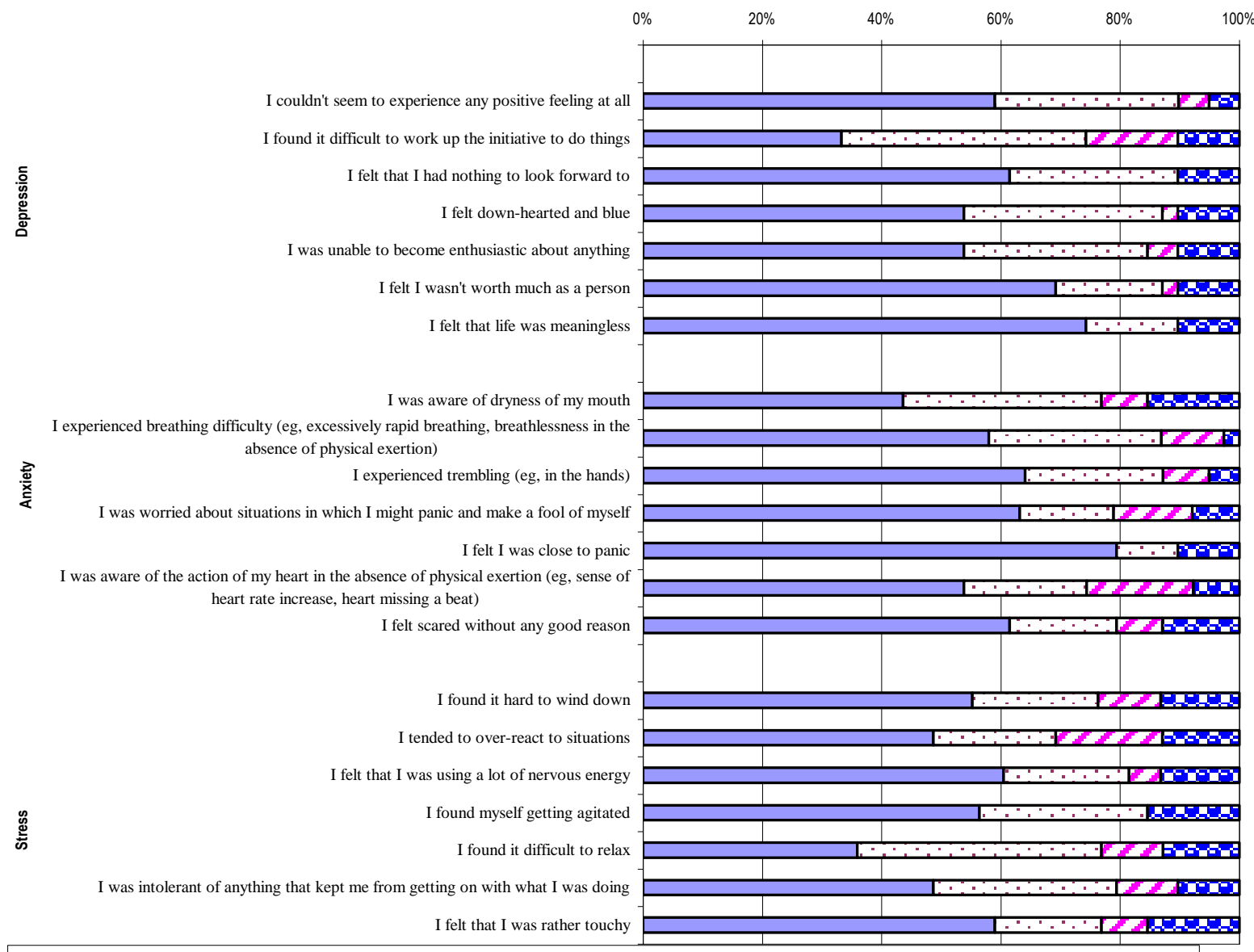

$\square$ Did not apply to me at all

$\square$ Applied to me to some degree or some of the time

$\square$ Applied to me a considerable degree or a good part of the time $\boldsymbol{\square}$ Applied to me very much or most of the time 
Figure 2. Levels of Depression, Anxiety, and Stress

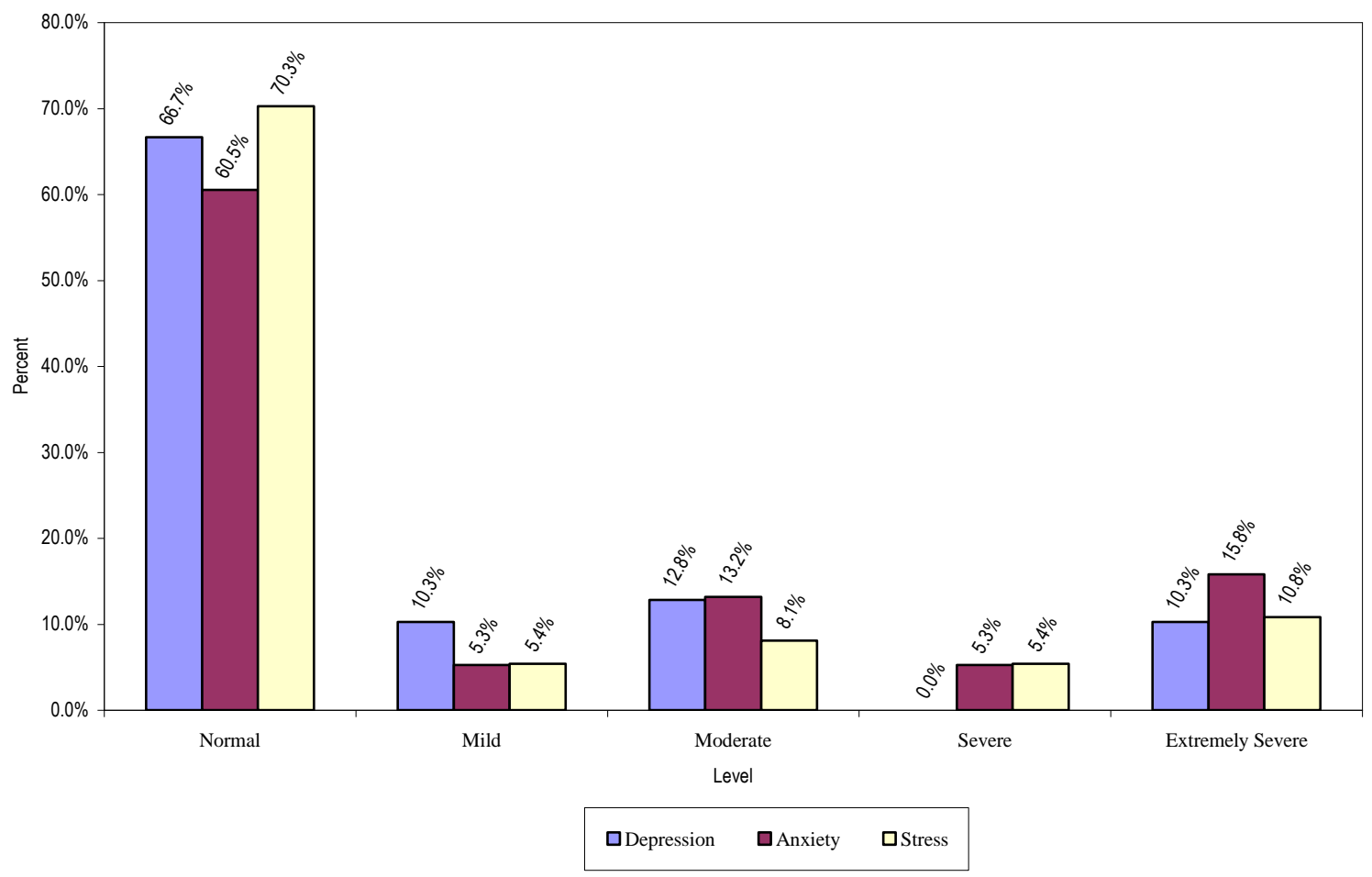

